\title{
Evaluating the efficacy and safety of a novel endo- scopic fluorescence imaging modality using oral 5-aminolevulinic acid for colorectal tumors
}

Authors

Institutions
Eriko So Tsuruki', Yutaka Saito ${ }^{1}$, Seiichiro Abe ${ }^{1}$, Hiroyuki Takamaru' ${ }^{1}$, Masayoshi Yamada' ${ }^{1}$ Taku Sakamoto ${ }^{1}$, Takeshi Nakajima' ${ }^{1}$, Takahisa Matsuda' ${ }^{1}$, Shigeki Sekine ${ }^{2}$, Hirokazu Taniguchi ${ }^{2}$

${ }^{1}$ Endoscopy Division, National Cancer Center Hospital, Tokyo, Japan

${ }_{2}^{2}$ Pathology Division, National Cancer Center Hospital, Tokyo, Japan

\section{Bibliography}

Dol http://dx.doi.org/

10.1055/s-0041-110432

Published online: 11.1.2016

Endoscopy International Open 2016; 04: E30-E35

(c) Georg Thieme Verlag KG

Stuttgart · New York

E-ISSN 2196-9736

\section{Corresponding author}

Yutaka Saito, MD, PhD

National Cancer Center Hospital Division of Endoscopy

5-1-1 Tsukiji

Chuo-ku, Tokyo, 104-0045

Japan

Phone: +81-3-3542-2511

Fax: +81-3-3542-3815

ytsaito@ncc.go.jp
Background and study aims: Five-aminolevulinic acid (5-ALA) is being increasingly used for photodynamic diagnosis and therapy of various types of tumors including brain, urologic, and other neoplasias. The use of 5-ALA to treat Barrett's carcinomas has been documented, but its clinical effectiveness for diagnosis of gastrointestinal tumors, particularly early cancers, remains unknown.

Patients and methods: The aim of our feasibility study was to evaluate the visibility of colorectal tumors using endoscopic fluorescence imaging (EFI) after oral administration of 5-ALA. The lesions identified by direct visualization and by the spectrums produced using EFI modality with 5ALA were compared to the clinicopathologic features of resected specimens.

Results: Twenty-three patients with a total of 27 known colorectal lesions were enrolled in the

\section{Introduction}

Colonoscopy reduces mortality from colorectal cancer through detection and treatment of tumors at an earlier and more treatable stage, but lesions that are particularly flat and depressed lesions remain challenging to detect, even with advanced modalities such as chromoendoscopy, narrow band imaging (NBI), and autofluorescence imaging (AFI) [1-4]. Therefore, new modalities and methods should be entertained. We focused on 5-aminolevulinic acid (5-ALA) because it has attracted increasing interest for photodynamic diagnosis (PDD) and photodynamic therapy (PDT) in various types of cancers [5].

5-ALA is a new-generation photosensitive substance that is an endogenous natural amino acid produced primarily in mitochondria and a common precursor of porphyrins. The great advantage of 5-ALA over other photodynamic substances is its lack of photosensitization. Protoporphyrin IX (PpIX), which is synthesized from 5-ALA in study. The median tumor size was $30 \mathrm{~mm}$ (range 10 - 75). Eleven of the lesions were flat or depressed lesions and 16 were sessile. Red fluorescence was observed in 22 out of 27 lesions. Red fluorescence was negative in 4 out of 11 flat or depressed lesions. In comparison with histopathologic findings, the rates of red fluorescence visibility were $62.5 \%$ in low-grade intraepithelial neoplasia, $77.8 \%$ in high-grade neoplasia, and $100 \%$ in submucosal carcinoma. Red fluorescence visibility increased with the degree of dysplasia. There were no significant adverse events identified in this study.

Conclusions: This feasibility study using EFI with 5-ALA demonstrated high visibility of superficial colorectal neoplasia. EFI with 5-ALA appears to be a novel, safe technique for improving realtime colorectal tumor imaging.

mitochondria of humans, is an intrinsic and a suitable photosensitizer with few documented adverse effects. PpIX preferentially accumulates much more in tumor cells than in normal cells. When PpIX is excited by irradiation with a specific wavelength of light, mainly visible blue light, it emits red fluorescence.

Detecting this red fluorescence can lead to accurate identification of cancer. This is the reason that 5-ALA is useful in PDD. Oral administration of 5-ALA is simple, safe and, thus, more acceptable to patients. Clinical application of 5-ALA in the fields of brain [5] and urologic tumors [6] and other neoplastic lesions is progressing. Some reports revealed that 5-ALA was useful in the detection of Barrett's cancer [7], gastric cancer,[8] and high-grade dysplasia in inflammatory bowel disease [9]. However, its clinical effectiveness in colorectal tumors, particularly for flat neoplasias, remains unknown. Thus, we conducted a feasibility study using endoscopic fluorescence imaging 
(EFI) with 5-ALA to evaluate its efficacy and safety for imaging of colorectal tumors.

The aim of this study was to demonstrate the potential for imaging adenomas, flat lesions, and early colorectal cancers using EFI with oral administration of 5-ALA. We also wanted to investigate the acceptability to patients and safety of this novel technique.

\section{Patients and methods \\ $\nabla$}

Patients with known adenoma or early colorectal cancer diagnosed endoscopically at previous institutions who were referred to the National Cancer Center Hospital for endoscopic treatment or surgery were enrolled from December 2012 to August 2014. Patients with severe comorbidities and known photosensitivity were excluded. Those enrolled had varied macroscopic lesions, including flat and depressed tumors.

This study was approved by the institutional review boards (IRB) of the hospital. All patients provided written and verbal informed consent to undergo endoscopy and to participate in the study whose description was presented according to the IRB. Use of 5ALA is covered by universal health care in Japan as a diagnostic drug for malignant glioma, but not for gastrointestinal tumors. In the IRB, therefore, we applied for use of 5-ALA as an off-label diagnostic product.

\section{Preparation for this study}

Patients took oral 5 -ALA $(20 \mathrm{mg} / \mathrm{kg}$ ) dissolved in $50 \mathrm{~mL}$ water about 4 hours before EFI [9]. They were instructed to avoid direct sunlight for 48 hours after oral administration. Bowel preparation was conducted with 2000 to $3000 \mathrm{~mL}$ of polyethylene glycol (PEG).

\section{Modality of EFI}

A prototype endoscope, XG-0001 with Advancia electronic endoscope system (FUJIFILM, Tokyo, Japan), was used [10]. A small filter, which would shade the blue light (less than $405 \mathrm{~nm}$ ) off the screen, was placed in the scope in order to visualize red fluorescence distinctly. A new system called VLD (Violet Laser Diode)-EX (SBI pharmaceuticals Co., Ltd, Japan) ( Fig. 1 a) exposed the colorectal tumors to light at a wavelength of $410 \mathrm{~nm}$ by using a special probe-type fiber ( $\bullet$ Fig.1 b). VLD-EX also displayed a spectrum of PpIX in real time and calculated the relative intensity of the fluorescence.

For positive control, when a PpIX reagent was irradiated with the excitation light of this system, red fluorescence was observed ( $\bullet$ Fig.2a). Red fluorescence was revealed by a characteristic spectrum of PpIX, which had two primary wavelengths of 636 $\mathrm{nm}$ and $705 \mathrm{~nm}$. In this study, we focused on the sharp peak at $636 \mathrm{~nm}$, which was displayed in VLD-EX ( $\bullet$ Fig. 2 b). In addition, an alarm with detection sound was set off when the fluorescence intensity at $636 \mathrm{~nm}$ exceeded the trial threshold. The wavelength of background noise and autofluorescence was also displayed in the gray area of $\bullet$ Fig. $\mathbf{2 b}$.

\section{Endoscopic observation and treatment}

After inserting XG-0001 with white light and identifying the target lesion, we washed mucus on the tumor and surrounding mucosa carefully with water containing $1 \%$ Pronase MS $^{\circledR}$ (Kaken Pharmaceutical Co., Ltd, Japan) and then set the special fiber through the channel of the endoscope. We kept the tip of the spe-
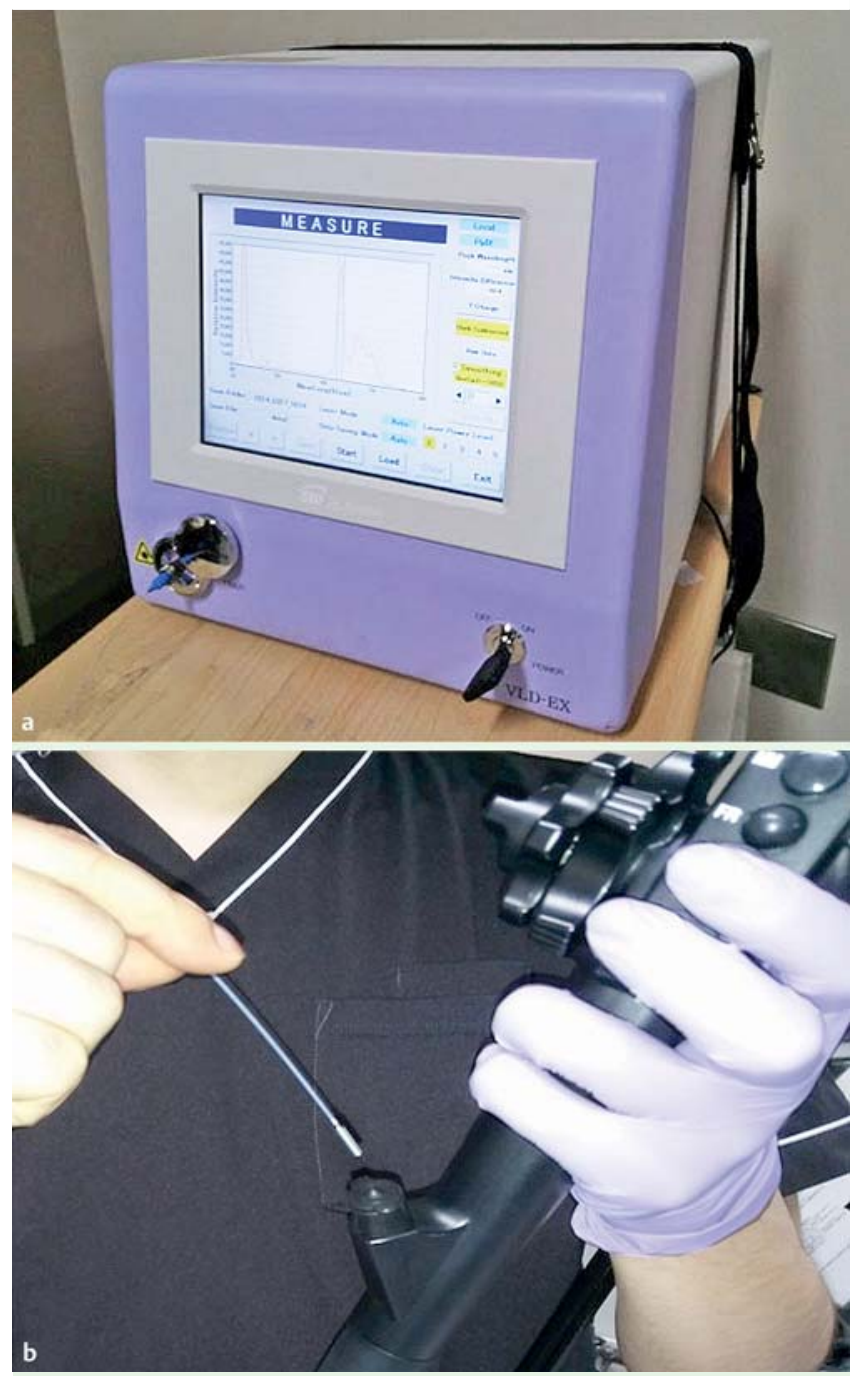

Fig. 1 a VLD-EX exposes the colorectal tumors to light using a special probe-type fiber. $\mathbf{b}$ Special probe-type fiber was inserted from the channel of the endoscope.

cial fiber at a distance of 1 to $2 \mathrm{~cm}$ from the lesion and dimmed the light of the endoscope. When we pressed the START button of VLD-EX, the lesion was irradiated with the excitation light from the special fiber. Because the irradiation area was small, we maneuvered the endoscope to observe the fluorescence imaging on the lesion. Two endoscopists judged the visibility of red fluorescence and validated the spectrum and the alarm from VLD-EX. When the FREEZE button of the VLD-EX was pressed, the irradiation stopped and the spectrum, which was displayed in the monitor, was recorded and stored in VLD-EX. As a control, normal mucosa near the lesion was observed in the same way. Generally, it took less than 10 minutes to observe fluorescence imaging. EFI and the variable spectrums were also recorded by OPERIO system (OLYMPUS, Tokyo, Japan).

Lesions were endoscopically diagnosed as adenoma (low-grade intraepithelial neoplasia), intramucosal cancer (Tis, high-grade neoplasia) or slightly invasive submucosal cancer (T1a, submucosal carcinoma) using magnifying endoscopy with NBI and chromoendoscopy. Such lesions were resected by endoscopic mucosal resection or endoscopic submucosal dissection as appropriate. For lesions endoscopically diagnosed as deep invasive submuco- 


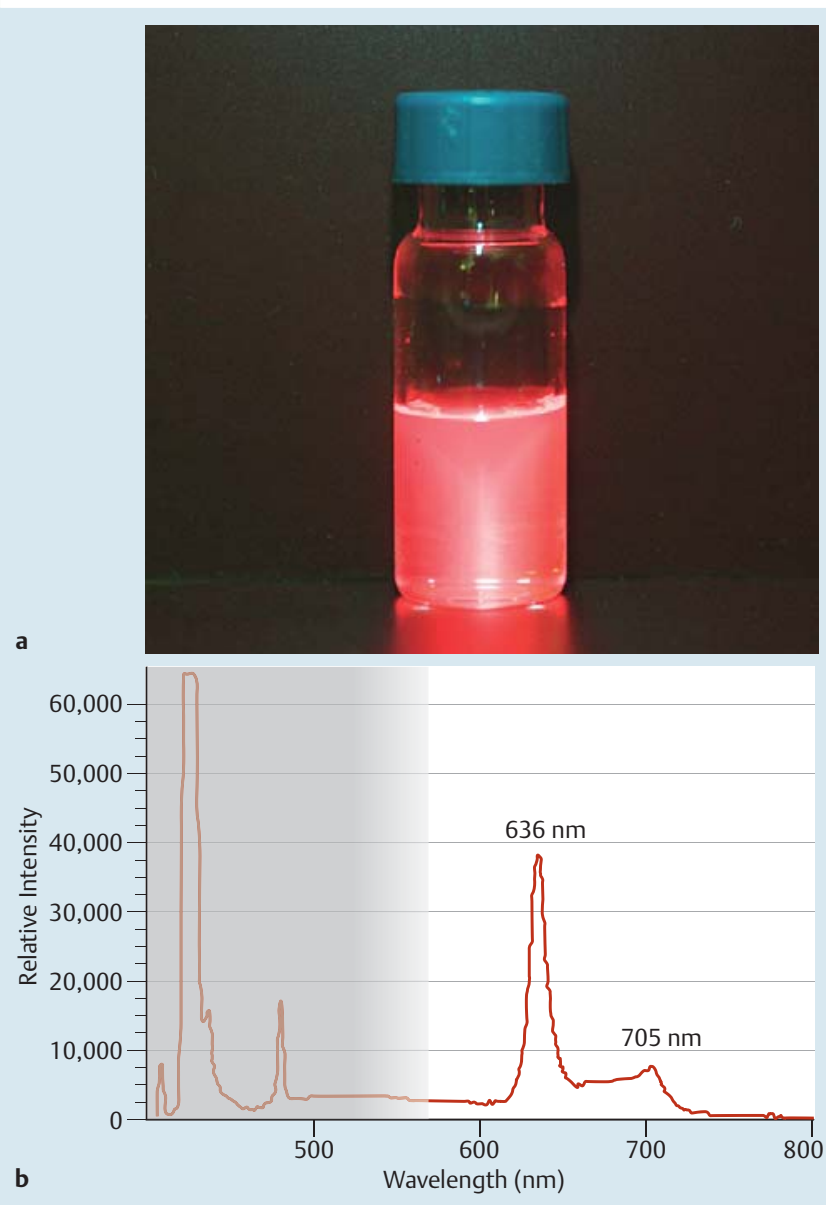

Fig. 2 a PpIX reagent for positive control. b Specific spectrum of PpIX is displayed in monitor of VLD-EX. Background noise and autofluorescence are displayed in the grey area. PpIX: Protoporphyrin IX.

sal cancer (T1b, submucosal carcinoma) or beyond, surgery was performed on another day.

\section{Histopathological Assessment}

The visible red fluorescence and the spectrum produced were compared with the histopathological features of resected specimens.

All specimens were evaluated after being cut into 2-mm slices and examined.

Experienced gastrointestinal pathologists assessed macroscopic type, histologic type, tumor size, depth of invasion, lymphovascular invasion, and resected margins, according to the Japanese Classification of Colorectal Carcinoma [11] and Paris endoscopy classification [12].

\section{Evaluation}

Visibility of red fluorescence

A positive red fluorescence meant that red fluorescence was observed in tumor ( $\bullet$ Fig.3a, Fluorescence + ). Typically the specific spectrum of PpIX was shown ( $\bullet$ Fig. $3 \mathbf{b}$ ). A negative red fluorescence meant lack of red fluorescence in imaging ( $\bullet$ Fig.3c, Fluorescence -), which indicated the absence of specific spectrum with a peak at $636 \mathrm{~nm}(\boldsymbol{F i g . 3 d})$.

\section{Intensity and alarm}

Calculated intensity of VLD-EX was a relative value. Baseline intensity was variable due to some accumulation of PpIX in normal gastrointestinal mucosa, inflammation, and other facts. Therefore, we used an intensity difference (ID $=\mathrm{I}_{636 \mathrm{~nm}}-\mathrm{I}_{626 \mathrm{~nm}}$ ) to evaluate the spectrum of VLD-EX ( $\bullet$ Fig.3b). Tentatively, the threshold of ID greater than 5000 was used to signify the specific spectrum of PpIX from tumors, and the alarm was set off when the threshold was exceeded. To check the adverse effects, blood tests were taken on the next day and 2 weeks after administration of 5-ALA. White blood cell count, hemoglobin, platelet, total bilirubin, aspartate transaminase, alanine transaminase, creatinine, blood urea nitrogen, and C-reactive protein were evaluated.

\section{Results \\ $\nabla$}

Twenty-three patients with 27 colorectal lesions were enrolled in this study.

Clinicopathologic features were as follows: male/female $=12 / 11$; median age 68 years (range $52-85$ ); location colon/rectum = $17 / 10$; median tumor size $30 \mathrm{~mm}$ (range 10-75); macroscopic type flat or depressed/sessile (with protrusion) $=11 / 16$; treatment endoscopy/surgery $=21 / 6$ and; low-grade intraepithelial neoplasia (IEN)/high-grade neoplasia/submucosal carcinoma = 8/9/10.

Red fluorescence was observed in 22 out of 27 lesions (81.5\%). - Table1 reveals the characteristic of fluorescence, positive or negative. In 8 out of 22 lesions, red fluorescence was observed partially or in spots of tumor. According to different macroscopic types, 4 out of 11 flat or depressed lesions proved negative for red fluorescence.

In comparison with histopathologic findings ( Table 2 ), the rates of red fluorescence visibility were $62.5 \%$ in low-grade IEN, $77.8 \%$ in high-grade neoplasia, and $100 \%$ in submucosal carcinoma. Red fluorescence visibility increased with the degree of dysplasia. In low-grade IEN and high-grade neoplasia, the positive rate of red fluorescence was higher in sessile lesions than in flat or depressed lesions ( $100 \%$ vs. $0 \%$ in low-grade IEN and $83.3 \%$ vs. $66.6 \%$ in high-grade neoplasia).

As a result, the time lags between administration of 5-ALA and EFI ranged from 3 to 7.5 hours, although there was no relation to the visibility of red fluorescence. Excluding the two cases in which the spectrum was not recorded because of mishandling of VLD-EX, the specific spectrum of PpIX or the spectrum with sharp peak at $636 \mathrm{~nm}$ was detected in all positive red fluorescence cases. The relative intensity of specific spectrum of PpIX and ID was variable. Regarding adverse events, 5 out of 23 patients had vomiting after PEG solution, and 2 patients experienced slight reddening of their skin. None of the exhibited signs of hepatotoxicity or nephrotoxicity.

\section{Video}

- Video 1 shows a surgery case with positive fluorescence. A protruded 0-Is + Ila lesion was located in the sigmoid colon, a tumor size was $30 \mathrm{~mm}$ in diameter. We detected red light within the target area. The specific spectrum was detected by VLD-EX. In contrast, red light was not observed in normal mucosa. 


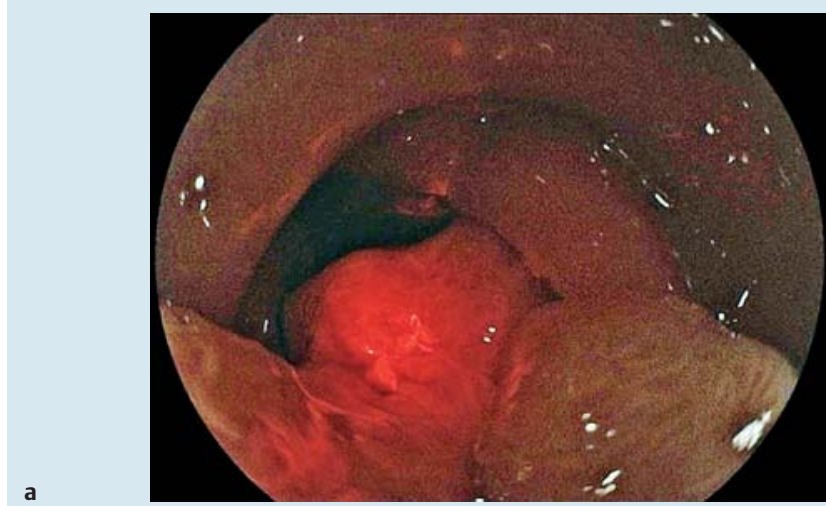

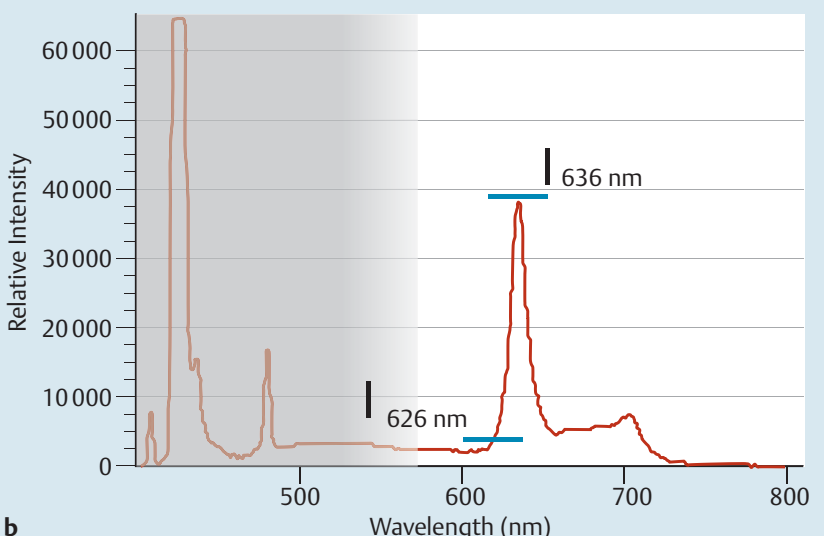

c

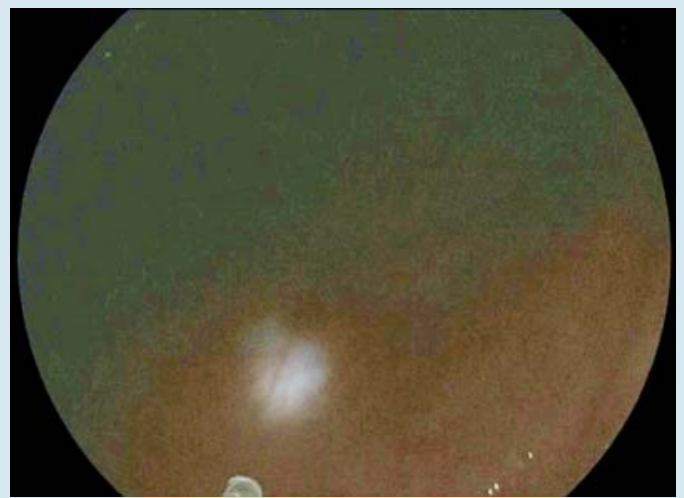

d

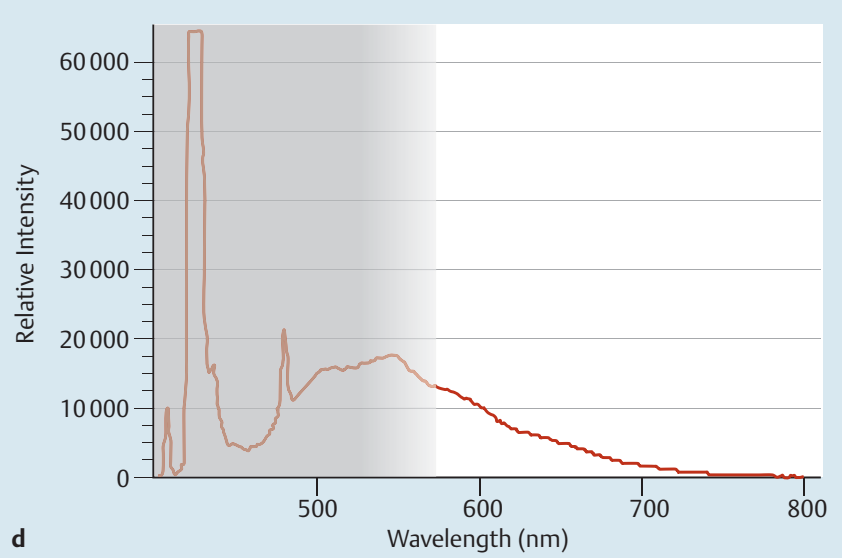

Fig. 3 a Fluorescence + b Specific spectrum of PPIX. Intensity Difference (ID): I ${ }_{636 \mathrm{~nm}}{ }^{-}{ }_{626 \mathrm{~nm}} I^{*}$ : Relative Intensity c Fluorescence d Non-specific spectrum.

\begin{tabular}{|lcc|} 
& $\begin{array}{l}\text { Fluorescence }+ \\
(\mathbf{n = 2 2 )}\end{array}$ & $\begin{array}{l}\text { Fluorescence - } \\
(\mathbf{n = 5 )}\end{array}$ \\
\hline Male/female & $11 / 11$ & $5 / 0$ \\
\hline Age, median (range) & $68(52-85)$ & $70(59-77)$ \\
\hline Location, colon/rectum & $12 / 10$ & $5 / 0$ \\
\hline Macroscopic type, Flat or depressed/sessile & $7 / 15$ & $4 / 1$ \\
\hline Size (mm), median (range) & $32.5(10-75)$ & $30(20-60)$ \\
\hline Low-grade IEN/high-grade neoplasia/submucosal carcinoma ${ }^{1}$ & $5 / 7 / 10$ & $3 / 2 / 0$ \\
\hline
\end{tabular}

Table 1 The characteristic of

fluorescence, positive or negative

IEN; intraepithelial neoplasia

${ }^{1}$ Paris endoscopy classification criteria

\begin{tabular}{|c|c|c|c|}
\hline & \multicolumn{3}{|l|}{ Histopathology } \\
\hline & Low-grade IEN & High-grade neoplasia & Submucosal carcinoma \\
\hline $\begin{array}{l}\text { Fluorescence }+ \\
\text { Total } 81.5 \%(22 / 27)\end{array}$ & $62.5 \%(5 / 8)$ & $77.8 \%(7 / 9)$ & $100 \%(10 / 10)$ \\
\hline Macroscopic type & & & \\
\hline Flat or depressed* & $0 \%(0 / 3)$ & $66.6 \%(2 / 3)$ & $100 \%(5 / 5)$ \\
\hline Sessile (with protrusion) ${ }^{1}$ & $100 \%(5 / 5)$ & $83.3 \%(5 / 6)$ & $100 \%(5 / 5)$ \\
\hline
\end{tabular}

Table 2 Fluorescence positive rate according to histopathology and macroscopic types

IEN; intraepithelial neoplasia

${ }^{1}$ Flat or Depressed: 0 -IIa, 0-Ila + Ilc, Sessile (with protrusion): 0-Is, 0-Is + Ila, 0-Is + IIc

\section{Discussion}

To the best of our knowledge, this is the first trial to demonstrate the clinical impact of orally ingested 5-ALA for photodynamic diagnosis of early-stage colorectal tumors in humans using real-life visualization with surgical pathology controls. Other molecular imaging probes such as heptapeptide [13] and nanospheres [14] have also been tested as novel imaging techniques for colorectal tumors. However, 5-ALA has already demonstrated benefits in both detection and treatment of other neoplasias. For that reason, we considered 5-ALA as our prime subject of interest for the detection of colorectal tumors. In addition, it has been demonstrated safe for clinical use in other neoplastic scenarios, providing a rationale reaffirming its use in colorectal tumors. 


\section{Red fluorescence visualization}

We demonstrated that red fluorescence could be visualized in most tumors and positively correlated with neoplastic stage. Red fluorescence visibility using EFI with 5-ALA was limited in flat or depressed lesions, given that it resulted in false negative results in 4 of 11 such cases. Red fluorescence visibility was recorded as "partially positive" in 8 lesions due to heterogeneity in appearance. In 4 lesions, which were combined flat and protruded, red fluorescence was identified in only the protruded areas. In the remaining 4 lesions, the red fluorescence was identified as patchy, lacking homogeneity, and thus not correlating fully with the clinicopathologic findings.

\section{VLD-EX spectrum analysis}

Because visibility of red fluorescence to the naked eye can be subjective, we used VLD-EX to aid the endoscopist's objective evaluation of the fluorescence because it incorporates a predetermined spectrum and an alarm system. The relative intensity of the specific spectrum of PpIX varied, however, because of three factors: (1) background noise with respect to VLD-EX itself; (2) autofluorescence of normal colonic mucosa and colonic tumor; and (3) inflammation resulting in accumulation of PpIX and baseline accumulation of PpIX by healthy colonic mucosa. As a result, the trial threshold of ID 5000 could not be confirmed in our sample size. Further examination is required to determine the threshold ID for the specific spectrum and alarm setting in the detection of particularly flat, dysplastic lesions such as nongranular laterally spreading tumor.

\section{5-ALA}

Detection of colorectal tumors in humans using HAL (Hexvix ${ }^{\circledR}$ Photocure, Oslo, Norway), a hexylester of 5-ALA, has been reported previously [15]. HAL may be more efficient at inducing PpIX formation than 5-ALA itself, but it cannot be ingested orally. Mayinger et al. reported that administration of HAL by enema induces selective lesion fluorescence and increases the rate of lesion detection in patients with colorectal neoplasia, especially those with flat, nonvisible adenomas [15].

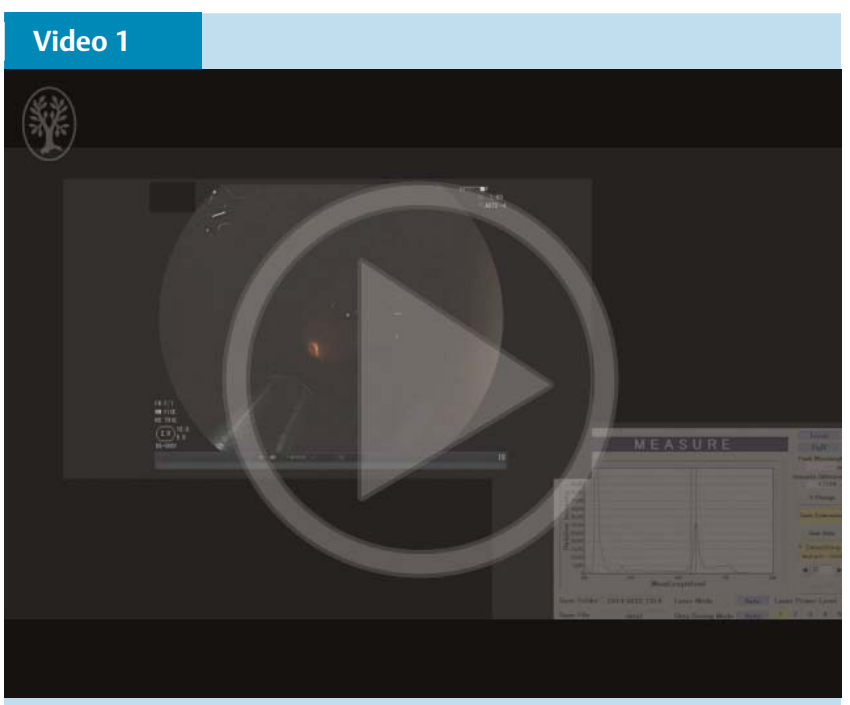

A positive fluorescence case. We detected red light within the lesion. The specific spectrum was detected by VLD-EX. Online content including video sequences viewable at: http://dx.doi.org/10.1055/s-0041-110432
In a previous case series using 5-ALA, Elena et al. reported on the fluorescence spectrum and the fluorescence intensity of malignant and premalignant colon lesions using their fluorescence spectroscopy system [16]. However, their study did not provide information on clinicopathologic details and the significant image of red fluorescence was not revealed in the reports.

The red fluorescence visibility in our study tended to be observed in submucosal carcinoma and in sessile adenomatous lesions with protrusion component.

Although the exact mechanisms of 5-ALA-induced PpIX preferential accumulation in cancer cells remain unclear, cellular proliferation, cell density, mitochondrial content, vascular proliferation, structural changes, and enzymatic downregulation (e.g. ferrochelatase) have been considered as contributing factors. Recent studies suggest that membrane transport of 5-ALA including the oligopeptide transporters (PEPT1 and 2) and ATP-binding cassette transporter G2 could be important factors as well [17].

This study was limited in that it was a feasibility study with a small sample, and because the irradiation area from the special fiber was small, the modality was still inadequate for detection of tumors in clinical practice. Improvement in devices is still necessary.

Already, some trials have demonstrated the potential for use of the technology to detect lymph node metastasis and peritoneal dissemination of pan-advanced colorectal cancers [18]. Therefore, 5-ALA fluorescence imaging may have a role in the future in detection of lymph node metastasis/peritoneal dissemination or select malignant potential of colorectal cancer.

\section{Conclusion}

$\nabla$

EFI with 5-ALA demonstrated high visibility of superficial colorectal neoplasia. It was interesting that red fluorescence visibility increased with the degree of dysplasia. EFI with 5-ALA appears to be a novel, safe technique for improving real-time colorectal tumor imaging, although examination of its use in more cases is needed.

\section{Competing interests: None}

\section{Acknowledgements}

$\nabla$

This study was financially supported in part by the National Cancer Center Research and Development Fund (23-B-17) and the Practical Research for Innovative Cancer Control from Japan Agency for Medical Research and development, AMED to Dr. Saito. We thank those who assisted with this study, namely, Chiko Sato, Hayato Sasaki, Masanori Sekiguchi, Nobuaki Ikezawa, and Hirohito Tanaka, Endoscopy Division, National Cancer Center Hospital. We thank Shraddha Gulati, Department of Gastroenterology, The Royal London Hospital and Klaus Monkemuller, the Basil I. Hirschowitz Endoscopic Center for Excellence, UAB for their editorial contributions. 


\section{References}

1 Zauber AG, Winawer SJ, O'Brien MJ et al. Colonoscopic polypectomy and long-term prevention of colorectal-cancer deaths. N Engl J Med 2012; 366: $687-696$

2 Rembacken BJ, Fujii T, Cairns A et al. Flat and depressed colonic neoplasms: a prospective study of 1000 colonoscopies in the UK. Lancet 2000; 355: $1211-1214$

3 Ikematsu H, Saito Y, Tanaka S et al. The impact of narrow band imaging for colon polyp detection: a multicenter randomized controlled trial by tandem colonoscopy. J Gastroenterol 2012; 47: 1099-1107

4 Matsuda T, Saito Y, Fu KI et al. Does autofluorescence imaging videoendoscopy system improve the colonoscopic polyp detection rate? -a pilot study Am J Gastroenterol 2008; 103: 1926-1932

5 Widhalm G, Wolfsberger S, Minchev G et al. 5-Aminolevulinic acid is a promising marker for detection of anaplastic foci in diffusely infiltrating gliomas with nonsignificant contrast enhancement. Cancer 2010; 15; 116: $061545-1552$

6 Ishizuka M, Abe F, Sano Y et al. Novel development of 5-aminolevulinic acid (ALA) in cancer diagnosis and therapy. Int Immunopharmacol 2011; 11: $358-365$

7 Endlicher E, Knuechel R, Hauser T et al. Endoscopic fluorescence detection of low and high grade dysplasia in Barrett's oesophagus using systemic or local 5-aminolaevulinic acid sensitisation. Gut 2001; 48: $314-319$

8 Namikawa T, Inoue K, Uemura $S$ et al. Photodynamic diagnosis using 5 aminolevulinic acid during gastrectomy for gastric cancer. J Surg Oncol 2014; 109: 213-217

9 Messmann H, Endlicher E, Freunek $G$ et al. Fluorescence endoscopy for the detection of low and high grade dysplasia in ulcerative colitis using systemic or local 5-aminolaevulinic acid sensitisation. Gut 2003; 52: $1003-1007$
10 Nakamura T, Oinuma T, Yamagishi $H$ et al. Evaluation of a novel highresolution magnifying videoendoscope that is capable of photodynamic diagnosis and therapy for gastric cancer. Photodiagnosis Photodyn Ther 2015; 12: $115-122$

11 Japanese Society for Cancer of the Colon and Rectum. Japanese Classification of Colorectal Carcinoma (English ed 2). Tokyo: Kanehara \& Co. Ltd; 2009

12 Participants in the Paris Workshop. The Paris endoscopic classification of superficialneoplastic lesions: esophagus, stomach, and colon. Gastrointestinal Endoscopy 2003; 58

13 Hsiung P, Hardy J, Friedland $S$ et al. Detection of colonic dysplasia in vivo using a targeted fluorescent septapeptide and confocal microendoscopy. Nat Med 2008; 14: 454-458

14 Kitamura T, Sakuma S, Shimosato $M$ et al. Specificity of lectin-immobilized f luorescent nanospheres for colorectal tumors in a mouse model which better resembles the clinical disease. Contrast. Media Mol Imaging 2015; 10: $135-143$

15 Mayinger B, Neumann F, Kastner $C$ et al. Hexaminolevulinate-induced fluorescence colonoscopy versus white light endoscopy for diagnosis of neoplastic lesions in the colon. Endoscopy 2010; 42: 28-33

16 Elena $V$, Andrey D, Antonina A et al. Fluorescence diagnostics of colon malignant and premalignant lesions using 5 -aminolevulinic acid. International Journal of Photoenergy 2014: Article ID 378673

17 Hagiya $Y$, Endo $Y$, Yonemura $Y$ et al. Pivotal roles of peptide transporter PEPT1 and ATP-binding cassette $(\mathrm{ABC})$ transporter ABCG2 in 5-aminolevulinic acid (ALA)-based photocytotoxicity of gastric cancer cells in vitro. Photodiagnosis Photodyn Ther 2012; 9: 204-214

18 Harada $K$, Harada $Y$, Beika $M$ et al. Detection of lymph node metastases in human colorectal cancer by using 5 -aminolevulinic acid-induced protoporphyrin IX fluorescence with spectral unmixing. Int J Mol Sci 2013; 21: 14(11): 23140-23152 